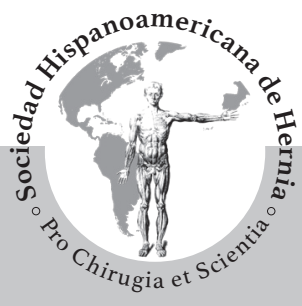

\title{
Hernia de Spiegel tras incisión de Pfannestiel: hipótesis etiopatogénica y revisión de la literatura
}

\section{Spiegel hernia after Pfannestiel incision: ethiopathogenic hypothesis and review of literature}

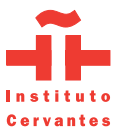

\section{Diego Flores Funes, Juan Antonio de la Torre Sánchez, José Aguilar Jiménez}

Servicio de Cirugía General. Hospital General Universitario Morales Meseguer. Murcia (España)

Recibido: 18-04-2018

Aceptado: 08-05-2018

Palabras clave:

Hernia de Spiegel, cesárea, etiología, cirugías previas.

\section{Resumen}

Introducción: La hernia de Spiegel supone un 1-2\% de todas las hernias de la pared abdominal. Existen factores de riesgo que predisponen a su aparición, entre los cuales se encuentran las cirugías abdominales previas.

Caso clínico: Presentamos un caso clínico de una paciente con una incisión de Pfannestiel previa por histerectomía, que terminó desarrollando una hernia de Spiegel al año siguiente de la cirugía.

Discusión: Se comenta su etiopatogenia, planteando la hipótesis del estiramiento y cizallamiento de la pared abdominal debido a la retracción producida por incisiones abdominales previas, embarazo y obesidad.

\section{Abstract}

Introduction: Spigelian Hernia accounts for 1-2 \% of all abdominal wall hernias. Some risk factors predispose to its appearance, such as previous abdominal surgery.

Case report: We present a clinical case of a patient with a previous hysterectomy by Pfannestiel incision, which ended in a Spigelian hernia the year after surgery.

Discussion: We also discuss its etiopathogenesis, posing the hypothesis of stretching and shearing of the abdominal wall due to the retraction produced by previous abdominal incisions, pregnancy and obesity.

* Autor para correspondencia: Diego Flores Funes. Servicio de Cirugía General. Hospital General Universitario Morales Meseguer. Avda. Marqués de los Vélez, s/n. 30008 Murcia (España)

Correo electrónico: diego.ff90@gmail.com 


\section{Introducción}

La hernia de Spiegel o hernia Spigeliana (HS) es una patología infrecuente, suponiendo aproximadamente un 1-2\% de todas las hernias de la pared abdominal ${ }^{1,2}$. No está descrita una etiología específica para este tipo de hernia, si bien existen factores de riesgo que predisponen a su aparición, tales como la obesidad y la pérdida rápida de peso, embarazos múltiples, EPOC, y cirugías previas, sobre todo laparoscópica ${ }^{3}$. Este último factor, aunque poco descrito, podría ser un factor causal de la aparición de la HS.

En este trabajo se presenta un caso clínico de una paciente con una incisión de Pfannestiel previa por histerectomía, que terminó desarrollando una HS en el año siguiente a la cirugía. También se realiza una discusión sobre la posible etiopatogenia de este tipo específico de HS.

\section{Caso clínico}

Mujer de 68 años, con obesidad grado II, e intervenida de histerectomía mediante una incisión de Pfannestiel el año previo, consultó a los 6 meses de la cirugía por tumoración en fosa ilíaca derecha cercana a cicatriz (fig. 1). Se realizó ecografía de pared abdominal, donde se aprecia una HS de $12 \mathrm{~cm}$ de diámetro máximo. Se completó estudio con TC abdominal con reconstrucción tridimensional, donde se confirmó la presencia de una HS que traspasaba el plano muscular del músculo transverso y oblicuo interno y quedaba contenida por el músculo oblicuo externo. El defecto de la pared abdominal medía $65 \mathrm{~mm}$, y tenía un tamaño de 130 por 95 por $61 \mathrm{~mm}$ (longitudinal por transversal por anteroposterior), con contenido de íleon y ciego, sin evidencia de sufrimiento intestinal (fig. 2).

Tras los hallazgos descritos, y tras obtener el consentimiento informado por escrito de la paciente, se decidió cirugía programada. En la misma, se realizó una incisión de abdominoplastia que incorporaba la cicatriz del Pfannestiel previo. Se disecó la HS, que se encontraba cubierta por fascia del músculo oblicuo mayor debilitado (fig. 3). Se continuó con una apertura de fas-

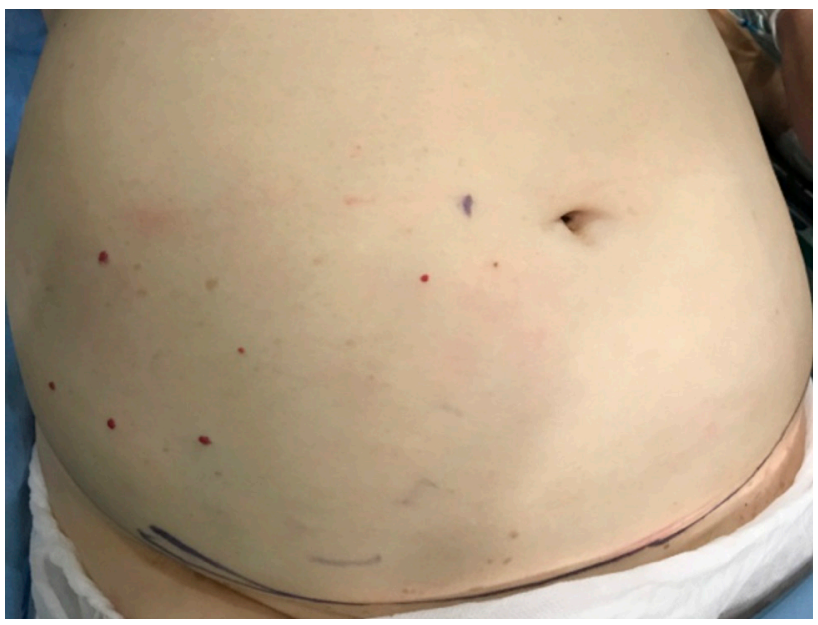

Figura 1. Fotografía de la pared abdominal anterior de la paciente, donde se aprecia tumoración en fosa ilíaca derecha.
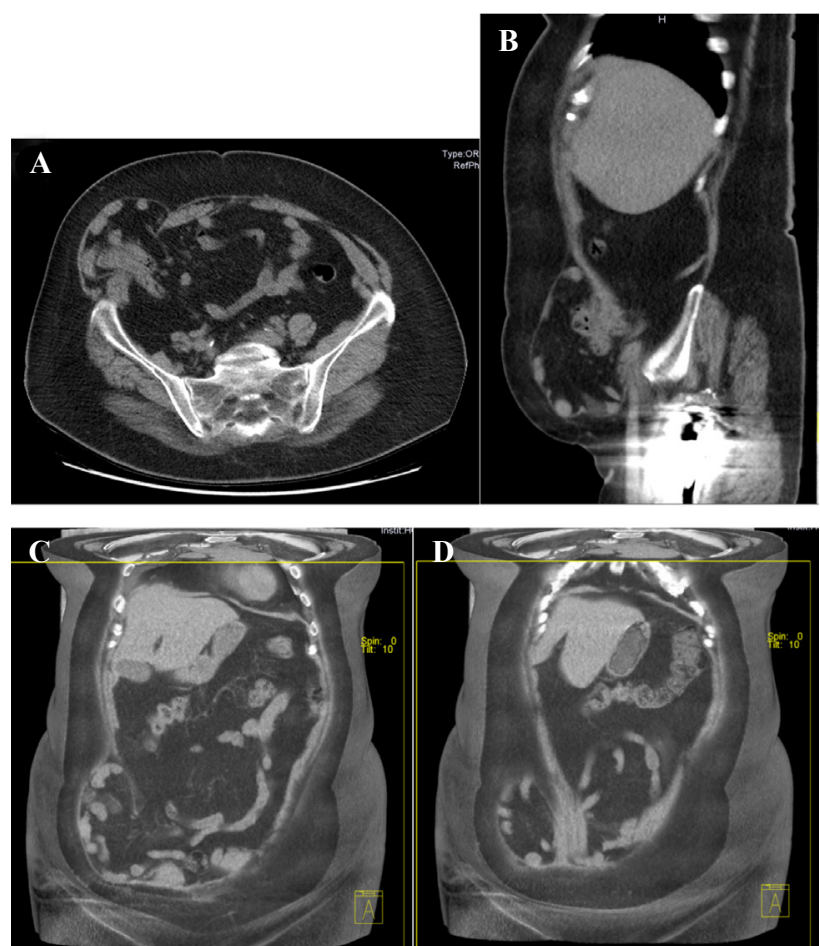

Figura 2. Tomografía computarizada con reconstrucción sagital, coronal y tridimensional. A. Corte axial de la HS. B. Corte sagital de la HS. C y D. Reconstrucción tridimensional en corte coronal de la HS.

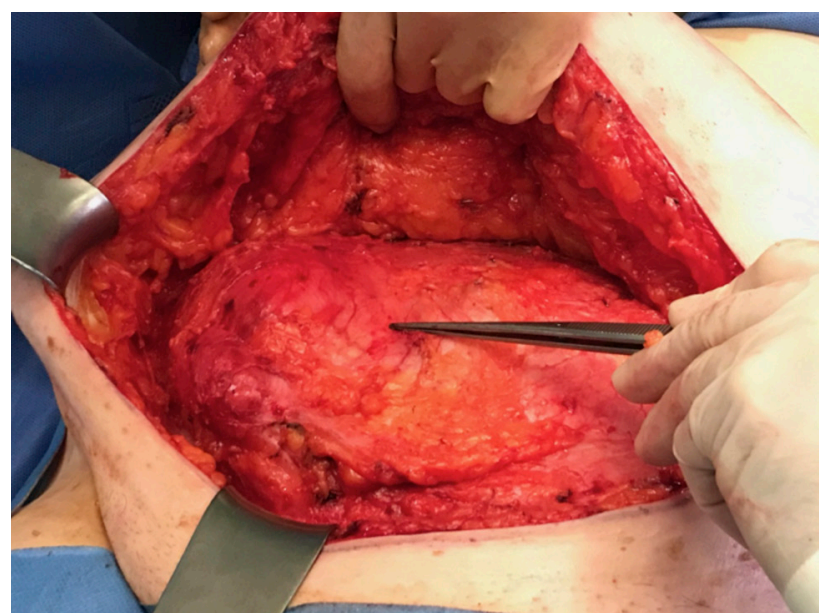

Figura 3. Aponeurosis del músculo oblicuo mayor debilitada que cubría la HS.

cia del músculo oblicuo mayor, disección del saco herniario (con anillo de $10 \mathrm{~cm}$ ) (fig. 4) y creación de un espacio preperitoneal. Se colocó una malla TiMESH ${ }^{\circ}$ de PFM (polipropileno revestido de titanio) de $15 \times 15$ anclada con puntos de polidioxanona (fig. 5). Se cerró la aponeurosis del músculo oblicuo mayor, quedando la malla completamente cubierta, y se completó la cirugía con una dermolipectomía parcial movilizando la inserción umbilical pero sin onfaloplastia. Se dejaron dos drenajes aspirativos y un vendaje compresivo. 


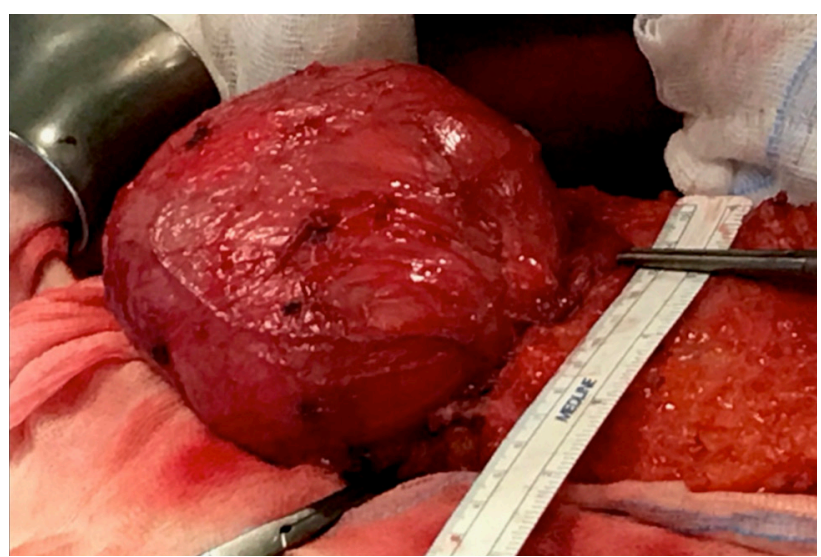

Figura 4. Saco herniario disecado completamente, con un tamaño del cuello de $10 \mathrm{~cm}$.

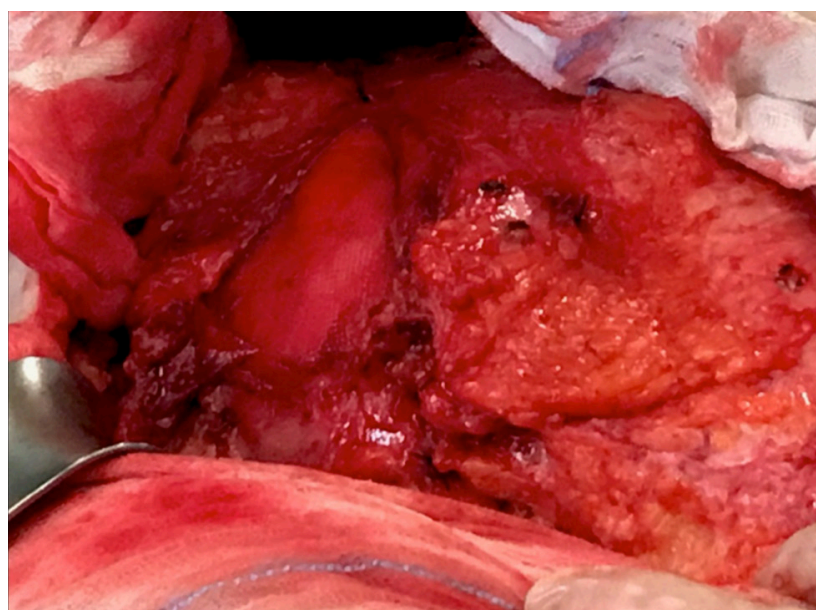

Figura 5. Colocación y fijación de malla TiMESH$^{\circ}$ en el espacio preperitoneal.

La paciente permaneció hospitalizada durante 5 días, en los que permaneció estable, con dolor bien controlado con analgesia, el abdomen y la herida sin signos de complicación, pudiendo retirar los drenajes previamente al alta. La paciente fue revisada en consulta externa un mes tras la cirugía, sin hallar complicaciones ni signos de recidiva herniaria.

\section{Discusión}

La hernia de Spiegel (HS), llamada así por la línea semilunar nominada por el anatomista Adriaan van Spiegel, fue descrita como una hernia ventral espontánea de aparición en dicha región por Klinkosch en $1764^{4}$. Su etiopatogenia no está completamente aclarada; si bien las HS congénitas asociadas a criptorquidia podrían producirse como una variante de ectopia testis, las producidas en el adulto no tienen un factor causal definido. Sí que se han observado una serie de factores de riesgo que influyen en su aparición. Uno de ellos es el embarazo: en este trabajo retrospectivo, se observó un $0.08 \%$ de incidencia de hernia umbilical, y un $0.12 \%$ de hernia inguinal durante la gestación ${ }^{5,6}$. Ello podría deberse a la rápida ganancia y pérdida de peso que distiende los tejidos de la pared abdominal y que durante el proceso podría debilitarlos. En el caso descrito en este trabajo, la paciente tuvo embarazos previos, lo que pudo haber influido en la aparición de la HS.

Otro factor importante es la cirugía previa: varias publicaciones describieron la relación de aparición de HS con intervenciones quirúrgicas anteriores, mayoritariamente por vía laparoscópica ${ }^{3,7}$. En este trabajo, en el que describen una serie de 201 pacientes intervenidos por vía laparoscópica, encontraron un $2 \%$ de HS, mientras que en este caso clínico los autores sugirieron que hasta en el $50 \%$ de los casos de hernia de Spiegel existen antecedentes de cirugía abdominal previa ${ }^{8,9}$. La explicación puede ser doble: en primer lugar, el traumatismo directo de la inserción del trocar de laparoscopia o de la incisión quirúrgica si se realiza vía abierta, puede condicionar la formación de una eventración a ese nivel, sobre todo si se realiza en una zona débil como la línea semilunar. Y, en segundo lugar, planteamos la hipótesis del estiramiento de la pared abdominal condicionado por un cierre abdominal previo: al realizar un cierre primario con una sutura con tensión, en su cicatrización se produce una retracción local en la zona de la incisión. Esto condiciona un estiramiento crónico de la pared abdominal cercana, que en el caso de tener una debilidad intrínseca (la línea semilunar de Spiegel, la región inguinal o umbilical), podría llegar su cizallamiento y formación de una hernia. Esta es la teoría que los autores consideramos que ha ocurrido con la paciente descrita, dado que tenía una cesárea previa, y la hernia que padecía se encontraba superior a dicha incisión, sin aparente relación con la misma. En esta serie una de sus pacientes fue una mujer histerectomizada en la que la hernia se encuentra lateral a la cicatriz, pudiendo explicarse también por esta teoría fisiopatológica, como ocurre en nuestro caso ${ }^{10-12}$.

Esta hipótesis es apoyada por la relación existente entre la aparición de una HS y otras hernias sincrónicas: se estima que entre el 23 y el $38 \%$ de los casos de HS (evidenciados en nuestra serie previa publicada) tienen otra hernia de pared abdominal ${ }^{1,10}$. La teoría fisiopatológica de esta relación podría ser la misma que la expuesta previamente: las incisiones previas condicionan una debilidad generalizada de la pared abdominal que podría influir en la aparición de hernias en las zonas débiles. Un ejemplo es la posible relación entre una incisión de Mc Burney o Rockey-Davis para una apendicectomía y la aparición de hernia inguinal derecha, aunque no hallamos estudios que corroboren dicha relación.

En este estudio reciente que incluye 107 pacientes, la serie más larga publicada hasta la fecha, proponen 3 estadios de HS: estadio I, sin saco peritoneal, únicamente con contenido preperitoneal, estadio II, con defecto y saco peritoneal menor de $5 \mathrm{~cm}$, que se pueden tratar laparoscópicamente, o estadio III, con orificio mayor de $5 \mathrm{~cm}$, que se prefiere un abordaje abierto para reconstruir la pared abdominal. Dos tercios de los casos fueron estadios II y III, siendo la paciente descrita en este trabajo un estadio III (orificio de $10 \mathrm{~cm})^{2}$.

La HS se encuentra actualmente infradiagnosticada: en este trabajo un $9 \%$ de los casos se hallaron de manera incidental y un $17 \%$ se descubrieron debido a su incarceración o estrangulación requiriendo cirugía urgente ${ }^{2}$. Por lo general, la presentación clínica es un dolor y tumoración a nivel de la línea semilunar que, dada la baja prevalencia de este tipo de hernias, lleva al clínico a confusión, sobre todo con lipomas abdominales y neuralgias cró- 
nicas. Por ello, ante dicha clínica, la primera prueba a realizar es la ecografía de pared abdominal, que nos permite distinguir entre una hernia u otro tipo de tumoración. Tras confirmar el diagnóstico, y para planificar una cirugía, la prueba prínceps es la TC, preferiblemente con reconstrucción tridimensional. En ella se puede observar el contenido herniario y la extensión exacta del orificio y del saco herniario. En este estudio, un $60 \%$ de los casos contenían grasa preperitoneal, un $20 \%$ contenían epiplón, un $74 \%$ contenían intestino delgado y un $31 \%$ intestino grueso ${ }^{11,12}$. En el caso presentado en este trabajo la TC fue fundamental, ya que se trataba de una hernia de gran tamaño y permitió conocer el contenido del saco herniario de cara a la cirugía.

En lo referente al tratamiento, la tendencia actual es intentar abordar el máximo número de pacientes posible mediante laparoscopia, preferiblemente mediante técnica intraabdominal (IPOM por sus siglas en inglés). En nuestra serie antigua, comparamos pacientes con cirugía abierta o cirugía laparoscópica, encontramos menor morbilidad y estancia hospitalaria en el grupo de laparoscopia. No existen ensayos aleatorizados entre las diferentes modalidades de cirugía laparoscópica para el tratamiento de la HS, pero en esta revisión de 2016 abogan por la técnica intraperitoneal como la más frecuente y con buenos resultados ${ }^{13-15}$. En nuestra serie posterior de 16 casos en la que se compararon los diferentes abordajes laparoscópicos, no encontramos diferencias significativas en tiempo operatorio ni en aparición de complicaciones, pero sí en costes, siendo $960 €$ más barata la IPOM, lo que apoya que dicho abordaje sea la vía de elección de la cirugía de la $\mathrm{HS}^{10}$.

Sin embargo, en el estudio de Webber et al. ${ }^{2}$, al proponer los 3 estadios de HS descritos anteriormente, recomiendan que en el estadio I el tratamiento debería ser la cirugía abierta, en el estadio II, sí que se puede tratar por vía laparoscópica, y en el estadio III, al tener un orificio herniario mayor de $5 \mathrm{~cm}$, se prefiere un abordaje abierto para reconstruir la pared abdominal. En este trabajo, en el que utilizan la técnica abierta de Litchestein entre los músculos oblicuos (no en el espacio preperitoneal), no encuentran recidivas ni complicaciones mayores, abogando por ser la técnica estándar ${ }^{13}$. Al ser nuestro caso un estadio III, optamos por el abordaje abierto, para asegurar una buena disección de los tejidos y una reconstrucción completa de la pared abdominal. Pero sin duda son necesarios más estudios, preferiblemente ensayos clínicos aleatorizados, para poder definir la vía de abordaje más indicada.

Como conclusión, cabe recalcar que la HS es una hernia poco frecuente, cuyo tratamiento es fundamentalmente mediante abordaje laparoscópico intraabdominal, aunque según el estadio se propone un tipo de cirugía u otra. La etiopatogenia podría estar en relación con el estiramiento y cizallamiento de la pared abdominal debido a la retracción producida por incisiones abdominales previas, al embarazo y a la obesidad. Esta teoría debe ser corroborada por estudios, preferentemente prospectivos, aunque la baja prevalencia y el largo período de seguimiento necesario hacen logísticamente difícil su realización.

\section{Bibliografía}

1. Ruiz de la Hermosa A, Amunategui Prats I, Machado Liendo P, Nevarez Noboa F, Muñoz Calero A. Spigelian hernia. Personal experience and review of the literature. Rev Esp Enferm Dig. 2010;102(10):583-6.

2. Webber V, Low C, Skipworth RJE, Kumar S, de Beaux AC, Tulloh B. Contemporary thoughts on the management of Spigelian hernia. Hernia. 2017;21(3):355-61.

3. Ussia A, Imperato F, Schindler L, Wattiez A, Koninckx PR. Spigelian hernia in gynaecology. Gynecol Surg. 2017;14(1):8-11.

4. Klinkosch JT. Programma Quo Divisionem Herniarum, Novumque Herniae Ventralis Specium Proponit. Rotterdam: Benam; 1764.

5. Jones BC, Hutson JM. The syndrome of Spigelian hernia and cryptorchidism: A review of paediatric literature. J Pediatr Surg. 2015;50(2):325-30.

6. Oma E, Bay-Nielsen M, Jensen KK, Jorgensen LN, Pinborg A, Bisgaard T. Primary ventral or groin hernia in pregnancy: a cohort study of 20,714 women. Hernia. 2017;21(3):335-9.

7. Bassi A, Tulandi T. Small Bowel Herniation Through a Spigelian Defect Within 48 Hours After Laparoscopy. J Minim Invasive Gynecol. 2013;20(3):392-3.

8. Paajanen H, Ojala S, Virkkunen A. Incidence of occult inguinal and Spigelian hernias during laparoscopy of other reasons. Surgery. 2006;140(1):9-12.

9. Kiliç MÖ, Değirmencioğlu G, Dener C. A rare case of Spigelian hernia combined with direct and indirect inguinal hernias. Turkish J Surg. 2017;33(1):40-2.

10. Moreno-Egea A, Campillo-Soto Á, Morales-Cuenca G. Which should be the gold standard laparoscopic technique for handling Spigelian hernias? Surg Endosc Other Interv Tech. 2015;29(4):85662.

11. Martin M, Paquette B, Badet N, Sheppard F, Aubry S, Delabrousse E. Spigelian hernia: CT findings and clinical relevance. Abdom Imaging. 2013;38(2):260-4.

12. Smereczyński A, Kołaczyk K, Lubiński J, Bojko S, Gałdyńska M, Bernatowicz E. Sonographic imaging of Spigelian hernias. J Ultrason. 2012;12(50):269-75.

13. Celdrán A, Señaris J, Mañas J, Frieyro O. The open mesh repair of Spigelian hernia. Am J Surg. 2007;193(1):111-3.

14. Moreno-Egea A, Carrasco L, Girela E, Martín J-G, Aguayo JL, Canteras M. Open vs Laparoscopic Repair of Spigelian Hernia. Arch Surg. 2002;137(11).

15. Barnes TG, McWhinnie DL. Laparoscopic Spigelian Hernia Repair. Surg Laparosc Endosc Percutan Tech. 2016;26(4):265-70. 